\title{
A Preliminary Comparative Assessment of the Role of CD8+ T Cells in Chronic Fatigue Syndrome/Myalgic Encephalomyelitis and Multiple Sclerosis
}

\author{
Ekua W. Brenu, ${ }^{1}$ Simon Broadley, ${ }^{2}$ Thao Nguyen, ${ }^{1,3}$ Samantha Johnston, ${ }^{1,3}$ \\ Sandra Ramos, ${ }^{1}$ Don Staines, ${ }^{1}$ and Sonya Marshall-Gradisnik ${ }^{1,3}$ \\ ${ }^{1}$ The National Centre for Neuroimmunology and Emerging Diseases, Griffith Health Institute, Griffith University, Gold Coast, Australia \\ ${ }^{2}$ School of Medicine, Griffith University, Gold Coast, Australia \\ ${ }^{3}$ School of Medical Science, Griffith University, Gold Coast, Australia
}

Correspondence should be addressed to Sonya Marshall-Gradisnik; s.marshall-gradisnik@griffith.edu.au

Received 6 October 2015; Revised 7 December 2015; Accepted 15 December 2015

Academic Editor: Jacek Tabarkiewicz

Copyright (C) 2016 Ekua W. Brenu et al. This is an open access article distributed under the Creative Commons Attribution License, which permits unrestricted use, distribution, and reproduction in any medium, provided the original work is properly cited.

Background. CD8+ T cells have putative roles in the regulation of adaptive immune responses during infection. The purpose of this paper is to compare the status of CD8+ T cells in Multiple Sclerosis (MS) and Chronic Fatigue Syndrome/Myalgic Encephalomyelitis (CFS/ME). Methods. This preliminary investigation comprised $23 \mathrm{CFS} / \mathrm{ME}$ patients, 11 untreated MS patients, and 30 nonfatigued controls. Whole blood samples were collected from participants, stained with monoclonal antibodies, and analysed on the flow cytometer. Using the following CD markers, CD27 and CD45RA (CD45 exon isoform 4), CD8+ T cells were divided into naïve, central memory (CM), effector memory CD45RA- (EM), and effector memory CD45RA+ (EMRA) cells. Results. Surface expressions of BTLA, CD127, and CD49/CD29 were increased on subsets of CD8+ T cells from MS patients. In the CFS/ME patients CD127 was significantly decreased on all subsets of CD8+ T cells in comparison to the nonfatigued controls. PSGL-1 was significantly reduced in the CFS/ME patients in comparison to the nonfatigued controls. Conclusions. The results suggest significant deficits in the expression of receptors and adhesion molecules on subsets of CD8+ T cells in both MS and CFS/ME patients. These deficits reported may contribute to the pathogenesis of these diseases. However, larger sample size is warranted to confirm and support these encouraging preliminary findings.

\section{Background}

Chronic Fatigue Syndrome/Myalgic Encephalomyelitis (CFS/ $\mathrm{ME}$ ) is characterised by significant impairments in physical activity as a consequence of severe fatigue and other flu like symptoms. CFS/ME remains an unexplained disorder with substantial physiological impairments where diagnosis is based on self-report measures. Fatigue is present in other disorders, including Multiple Sclerosis (MS), where $70 \%$ of patients are plagued with fatigue and this can be disabling in a minority of patients [1-3]. This fatigue can be severe and has consequences on normal daily activity $[4,5]$. CFS/ME has been reported in some MS patients where patients demonstrate classic CFS/ME symptoms such as intermittent headaches, malaise, and joint and muscle pain [6]. Nonetheless, diagnosis of MS also relies upon the demonstration of dissemination in time and space of central nervous system (CNS) lesions clinically or radiographically, often supported by biochemical and electrophysiological tests [7]. MS patients may be classified as relapsing remitting (RRMS), primary-progressive (PP-MS), secondary-progressive (SP-MS), or clinically isolated syndrome [8].

MS is a neurological disorder characterised by inflammatory demyelination in the CNS while CFS/ME patients may experience nervous system manifestations of lack of concentration and autonomic symptoms [9-12]. Neuroimaging studies have suggested the presence of neuroinflammation in the midbrain of CFS/ME patients [11]. Despite some relative ambiguities in CFS/ME symptomatology, immune dysregulation is a common occurrence in CFS/ME as well as 
in MS. It has previously been postulated that symptom similarities between CFS/ME and MS may be explained by shared neuroimmune pathways [13]. The most common immune abnormality in both CFS/ME and MS is related to Natural Killer (NK) cell cytotoxic activity. Dysfunctional NK cell activity has been reported in patients with MS and CFS/ME. In MS patients, alterations in NK cell function and receptor expression contribute significantly to the pathogenesis of the disease $[14,15]$. In CFS/ME, NK cell dysfunction involves decreased cytotoxic activity [16-18]. T cells have also been implicated in MS. Importantly, regulatory T cells (Tregs) are dysregulated and levels of these cells are related to the phase of the disease $[19,20]$.

Expansion of autoreactive lymphocytes in MS results in inflammatory and active immune responses in the CNS as these lymphocytes are able to migrate to the CNS and induce damage [21]. Reducing the activities of these autoreactive lymphocytes is the fundamental goal of many therapeutic interventions in MS [22]. CD8+ T cells have been extensively studied in MS owing to their presence in the CNS lesions of MS patients. In MS, pathogenic CD8+ T cells may induce proinflammatory reactions via interleukin- (IL-) 17 and interferon- (IFN-) $\gamma$ and eliminate oligodendrocytes while regulatory CD8+ T cells suppress autoreactive CD4+ T cells reactions and promote anti-inflammatory reactions [23]. In CFS/ME patients CD8+ $\mathrm{T}$ cells may display diminished levels of activation, reduced cytotoxicity, and low numbers of effector memory cells $[18,24]$.

It is apparent that CD8+ $\mathrm{T}$ cells are involved in the pathogenesis of CFS/ME and MS; hence, the aim of this study was to determine whether dysregulation in cytotoxic CD8+ T cells follows a similar pattern in CFS/ME and MS.

\section{Methods}

2.1. Subjects. CFS/ME participants were defined according to the International Consensus Criteria (ICC) [25]. Disability in the CFS/ME patients was measured using Dr. Bell's Disability Adjustment scale [26]. MS cases were clinically diagnosed as having MS according to the revised McDonald criteria [7]. MS disease progression and responsiveness were assessed using the Expanded Disability Status Scale (EDSS) [27] and disease severity was measured using the MS Severity Scale (MSSS) [7]. Nonfatigued controls had no incidence of $\mathrm{CFS} / \mathrm{ME}$ or MS and were in good health without evidence of fatigue. Excluded from the study were smokers, pregnant woman, breastfeeding, or having been clinically diagnosed with any other major diseases. All subjects gave informed written consent to participate in the study and the study received ethical approval from the Griffith University Human Ethics Committee (MSC/18/13/HREC) prior to commencement.

2.2. Assessment of CD8+ T Cell Phenotypes. Whole blood $(10 \mathrm{~mL})$ was collected from all participants and analysed within 12 hours of collection. To identify subsets of CD8+ $\mathrm{T}$ cells at different stages of differentiation, samples were labelled with fluorochrome conjugated monoclonal antibodies, including CD3, CD8, CD27, and CD45RA (CD45 exon isoform 4). Cells were analysed on the Fortessa 2.0 (Becton Dickenson (BD) Biosciences, San Jose). For each CD8+ T cell assessment, forward and side scatter plots were used to determine the lymphocyte population. Cells of interest were identified from the lymphocyte population as cells expressing CD3+ and CD8+. The expression of cytokines, chemokine receptors, adhesion molecules, and migratory molecules on CD8+ T cells were also examined using the following markers: CCR5, CCR7, CXCR3, CD49d, CD29, CD18, CD11a, PSGL-1, and CD127. Glycoprotein, CD44, was also examined.

2.3. Assessment of CD8+ T Cell Receptors. Inhibitory receptors were measured in whole blood cells stained with monoclonal antibodies including KLRG1, LAG3, CTLA4, and BTLA. The expression patterns of these inhibitory receptors were examined on the CD8+ T cell phenotypes. Coexpression of these receptors was also assessed on subsets of CD8+ T cells.

2.4. Statistical Analysis. Statistical analyses were executed using SPSS (version 18.0, SPSS Inc., Chicago, USA) and Graph Pad Prism (version 6.0, Graph Pad Software, Inc., San Diego, USA). A test for normality was performed using the Kolmogorov-Smirnov tests. ANOVA was used to determine significance for normally distributed data while the independent sample Kruskal Wallis test was used as the nonparametric. Bonferroni analysis was used to assess significant parameter differences post hoc. Pearson chi square test was used to determine significant gender differences. $P$ values less than or equal to 0.05 were considered significant. The data is expressed as either median or mean \pm standard error of the mean (SEM).

\section{Results}

3.1. Subject Characteristics. The characteristics of the participants recruited in the study are outlined in Table 1. A number of the CFS/ME patients were taking a combination of different medications at the time of the study. These medications include anticholinergic $(n=1)$, antihistamine $(n=1)$, antidepressant $(n=10)$, blood pressure medication $(n=1)$, steroids $(n=2)$, anticonvulsants $(n=4)$, Benodiazepines $(n=1)$, opioid receptor antagonist $(n=1)$, asthma $(n=3)$, cardiotonic agent $(n=2)$, anti-inflammatory $(n=3)$, opioids $(n=2)$, opioid analgesics $(n=4)$, triptans $(n=1)$, proton pump inhibitors $(n=3)$, vitamins and supplements $(n=5)$, anticoagulants $(n=2)$, and laxatives $(n=1)$. Nine of the CFS/ME patients were on no medications at the time of the study. Mean disability in the CFS/ME cases was $47.14 \% \pm 2.20$ (SD) using Dr. Bell's Disability score and classifying CFS/ME as moderate CFS/ME patients as described [28] (Table 2).

MS patients were not on any immunomodulatory therapies during this study, nor had they taken these previously. Of the $11 \mathrm{MS}$ patients, there were relapsing-remitting $(n=4)$, secondary-progressive $(n=2)$, primary-progressive $(n=$ $2)$, and clinically isolated syndrome $(n=3)$ cases. The average number of relapses (ever) rate among the MS cases 
TABLE 1: Characteristics of participants and blood parameters.

\begin{tabular}{|c|c|c|c|c|c|c|c|}
\hline & \multirow[b]{2}{*}{$\mathrm{CFS} / \mathrm{ME}$} & \multirow[b]{2}{*}{ MS } & \multirow[b]{2}{*}{ Controls } & \multicolumn{4}{|c|}{$P$ value } \\
\hline & & & & Overall & $\begin{array}{c}\text { MS versus } \\
\text { CFS/ME }\end{array}$ & $\begin{array}{c}\text { MS versus } \\
\text { control }\end{array}$ & $\begin{array}{c}\text { CFS/ME versus } \\
\text { control }\end{array}$ \\
\hline Participants $(n)$ & 23 & 11 & 30 & & & & \\
\hline Age (years) & $49.0 \pm 2.5$ & $56.0 \pm 4.9$ & $53.5 \pm 2.2$ & 0.72 & $>0.99$ & $>0.99$ & $>0.99$ \\
\hline Females, $n(\%)$ & $17(73.9)$ & $10(90.9)$ & $19(63.3)$ & 0.44 & & & \\
\hline Haemoglobin $(\mathrm{g} / \mathrm{L})$ & $135.0 \pm 2.25$ & $136.0 \pm 2.90$ & $139.0 \pm 2.35$ & 0.24 & $>0.99$ & 0.89 & 0.32 \\
\hline White cell count $\left(\times 10^{9} / \mathrm{L}\right)$ & $5.60 \pm 0.32$ & $6.80 \pm 0.64$ & $6.00 \pm 0.25$ & $0.04^{*}$ & $0.04^{*}$ & 0.52 & 0.28 \\
\hline Platelets & $228.0 \pm 13.34$ & $264.0 \pm 15.80$ & $249.50 \pm 10.69$ & 0.76 & $>0.99$ & $>0.99$ & $>0.99$ \\
\hline Haematocrit (\%) & $0.41 \pm 0.01$ & $0.40 \pm 0.01$ & $0.41 \pm 0.01$ & 0.35 & $>0.99$ & $>0.99$ & 0.49 \\
\hline Red cell count $\left(\times 10^{12} / \mathrm{L}\right)$ & $4.49 \pm 0.07$ & $4.55 \pm 0.13$ & $4.61 \pm 0.07$ & 0.53 & $>0.99$ & $>0.99$ & 0.79 \\
\hline $\mathrm{MCV}(\mathrm{fL})$ & $89.17 \pm 0.69$ & $89.20 \pm 0.85$ & $89.53 \pm 0.68$ & 0.72 & $>0.99$ & $>0.99$ & $>0.99$ \\
\hline Neutrophils $\left(\times 10^{9} / \mathrm{L}\right)$ & $3.38 \pm 0.25$ & $4.20 \pm 0.49$ & $3.53 \pm 0.17$ & 0.39 & 0.53 & 0.82 & $>0.99$ \\
\hline Lymphocytes $\left(\times 10^{9} / \mathrm{L}\right)$ & $1.63 \pm 0.09$ & $2.30 \pm 0.23$ & $1.95 \pm 0.10$ & $0.001^{*}$ & $0.001^{*}$ & 0.13 & 0.11 \\
\hline Monocytes $\left(\times 10^{9} / \mathrm{L}\right)$ & $0.31 \pm 0.02$ & $0.45 \pm 0.03$ & $0.33 \pm 0.02$ & $0.0004^{*}$ & $0.002^{*}$ & $0.0004^{*}$ & $>0.99$ \\
\hline Eosinophils $\left(\times 10^{9} / \mathrm{L}\right)$ & $0.14 \pm 0.015$ & $0.21 \pm 0.03$ & $0.12 \pm 0.01$ & $0.02^{*}$ & 0.13 & $0.01^{*}$ & 0.91 \\
\hline Basophils $\left(\times 10^{9} / \mathrm{L}\right)$ & $0.02 \pm 0.004$ & $0.03 \pm 0.004$ & $0.02 \pm 0.003$ & 0.41 & 0.56 & $>0.99$ & $>0.99$ \\
\hline ESR (mm/Hr) & $10.50 \pm 2.59$ & $13.00 \pm 5.29$ & $10.00 \pm 1.91$ & 0.21 & $>0.99$ & 0.89 & 0.26 \\
\hline
\end{tabular}

Data is represented as mean $\pm \mathrm{SEM}$, where $*$ represents $P<0.05$.

TABLE 2: Clinical characteristics of CFS/ME and MS.

\begin{tabular}{lcc}
\hline & CFS/ME $(n=23$, mean \pm SD $)$ & MS $(n=11$, mean \pm SD $)$ \\
\hline Dr. Bell's Disability & $47.14 \% \pm 2.20$ & $2.41 \pm 0.79$ \\
Expanded Disability Status Scale & & $2.85 \pm 0.89$ \\
Multiple Sclerosis Severity Scale & & $n=4$ \\
Courses & & $n=2$ \\
(i) Relapsing-remitting & & $n=2$ \\
(ii) Secondary-progressive & & $n=3$ \\
(iii) Primary-progressive & & $6.11 \pm 2.45$ \\
(iv) Clinically isolated syndrome & $35.28 \pm 4.63$ & $13.76 \pm 3.83$ \\
Age of onset (years) & $14.96 \pm 8.87$ & $2.4 \pm 0.55$ \\
Duration (years) & & \\
Relapses rate & & \\
\hline
\end{tabular}

was $2.4 \pm 0.55$. MS mean age was $56.0 \pm 4.9(\mathrm{SD})$ years reported illness onset of an average of 6.11 years \pm 2.45 (SD) for a duration of 13.76 years \pm 3.83 (SD). The mean EDSS and MSSS scores for the MS patients were $2.41 \pm 0.79$ (SD) and $2.85 \pm 0.89$ (SD), respectively, which classifies MS patients as moderately disabled (Table 2).

In each group there were a large percentage of females in comparison to males but there was no significant difference in gender. Full blood count analyses were performed on all samples to determine the distribution of the different blood cells (Table 1). White blood cells, lymphocytes, monocytes, and eosinophils were significantly higher in the MS group compared to the other groups. CD8+ T cell phenotypes CD27 and CD45RA surface markers were used to determine lineage differentiation (naïve and memory phenotypes) of the CD8+ T cells. Four different subsets of CD8+ T cells were characterized including CD8+CD3+CD27+CD45RA+ cells (naïve), CD8+CD3+CD27+CD45RA- cells (central memory [2]), CD8+CD3+CD27-CD45RA- cells (effector memory $[\mathrm{EM}]$ ), and CD8+CD3+CD27-CD45RA+ cells (CD45RA+ effector memory [EMRA]). There were no significant differences in total CD8+ T cells, naïve, CM, EM, and EMRA CD8+ T cells among the three groups (Table 3 ).

3.1.1. Quantitation of Inhibitory Receptors on CD8+ T Cells. Surface expression of the following inhibitory receptors was examined on the CD8+ T cells, KLRG1, LAG3, CTLA4, and BTLA. These receptors were measured on total CD8+ $\mathrm{T}$ cells and subsets of CD8+ $\mathrm{T}$ cells at different stages of differentiation as previously described. Only BTLA was significantly elevated in the naïve and CM CD8+ T cells from the MS patients compared with the CFS/ME patients and the nonfatigued controls (Figure 1). 
TABLE 3: Distribution of total and subsets of CD8+ T cells in CFS/ME patients, MS patients, and nonfatigued controls.

\begin{tabular}{|c|c|c|c|c|c|c|c|}
\hline \multirow[b]{2}{*}{ CD8+ T cells (\%) } & \multirow[b]{2}{*}{ CFS/ME } & \multirow[b]{2}{*}{ MS } & \multirow[b]{2}{*}{ Nonfatigued controls } & \multicolumn{4}{|c|}{$P$ value } \\
\hline & & & & Overall & $\begin{array}{c}\text { MS versus } \\
\text { CFS/ME }\end{array}$ & $\begin{array}{c}\text { CFS/ME versus } \\
\text { control }\end{array}$ & $\begin{array}{c}\text { MS versus } \\
\text { control }\end{array}$ \\
\hline $\begin{array}{l}\text { Total } \\
\text { CD8+ T cells }\end{array}$ & $13.25 \pm 0.86$ & $12.36 \pm 1.89$ & $13.24 \pm 0.88$ & 0.84 & $>0.99$ & $>0.99$ & $>0.99$ \\
\hline $\begin{array}{l}\text { Naïve } \\
\text { CD8+ T cells }\end{array}$ & $33.86 \pm 3.89$ & $36.76 \pm 5.76$ & $24.20 \pm 2.19$ & 0.07 & $>0.99$ & 0.12 & 0.29 \\
\hline $\begin{array}{l}\text { CM } \\
\text { CD8+ T cells }\end{array}$ & $31.88 \pm 2.63$ & $38.50 \pm 2.36$ & $32.93 \pm 2.75$ & 0.51 & 0.81 & $>0.99$ & 0.88 \\
\hline $\begin{array}{l}\text { EM } \\
\text { CD8+ T cells }\end{array}$ & $12.52 \pm 2.15$ & $15.83 \pm 2.77$ & $18.64 \pm 2.13$ & 0.07 & $>0.99$ & 0.08 & 0.51 \\
\hline $\begin{array}{l}\text { EMRA } \\
\text { CD8+ T cells }\end{array}$ & $10.18 \pm 2.21$ & $6.86 \pm 2.22$ & $13.38 \pm 2.13$ & 0.06 & 0.82 & 0.43 & 0.08 \\
\hline
\end{tabular}

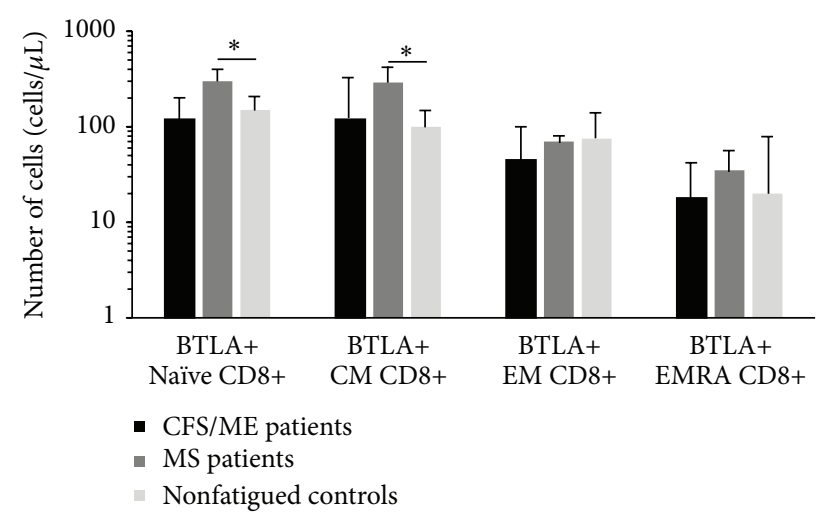

Figure 1: Expression of BTLA on CD8+ T cells in CFS/ME, MS, and nonfatigued controls. BTLA was increased on naïve and CM CD8+ $\mathrm{T}$ cells in the MS compared with controls. Data is represented as median \pm SEM, where $*$ represents $P<0.05$ (EM: effector memory, EMRA: effector memory RA, and CM: central memory).

3.1.2. Expression Pattern of Cytokine and Chemokine Receptors. Expression of cytokine receptors including CCR7, CCR5, and CD127 was measured on total CD8+ T cells and subsets of CD8+ T cells at different stages of differentiation. CD49/CD29 was significantly reduced on the EM CD8+ T cells of the CFS/ME patients in comparison to the nonfatigued controls, while in the MS patients, CD49d/CD29 was significantly elevated in the naïve and EMRA CD8+ T cells in comparison to the nonfatigued controls (Figure 2(a)). When the expressions of these receptors were examined, significant decrease in the expression of CD127+ was observed on most subsets of CD8+ T cells from the CFS/ME patients while in the MS patients, CD127 expression was reduced on naïve, EM, and EMRA subsets of CD8+ T cells in the CFS/ME patients (Figure 2(b)). Differential levels of integrins and selectins and cell surface glycoproteins PSGL, KLRG1, CD11a/CD18, and CD44 were measured on total and subsets of CD8+ T cells at different stages of differentiation. PSGL-1 was significantly reduced on EMRA CD8+ T cells in the CFS/ME patients in comparison to the nonfatigued controls (Figure 3 ).

\section{Discussion}

This preliminary study has identified significant impairments in subsets of CD8+ T cells in CFS/ME and MS patients. Overall the MS patients showed significant differences in the expression of receptors and adhesion molecules in comparison to the CFS/ME patients. These results demonstrate CD8+ $\mathrm{T}$ cells might play a role in the pathogenesis of MS compared with CFS/ME. PSGL-1 is elevated on CD4+ T cells in RRMS patients and this may suggest an important role in the transmigration of lymphocytes to the CNS [29]. However, total CD8+ T cells in these patients have stable levels of PSGL1 [29]. Therefore, the expression of PSGL-1 on CD8+ T cells may to some extent be dependent on the stage and type of disease. Nonetheless, it is possible that these cells have high affinity to transmigrate the blood brain barrier (BBB) and this may be specific to naïve CD8+ T cells in MS. In MS, PSGL1 is important for the adhesion and recruitment of CD8+ $\mathrm{T}$ cells to the inflamed CNS [30]. CD49d/CD29 represents the $\alpha 4 \beta 1$ integrin, adhesion molecules which are important during inflammation. CD49d/CD29 has been shown to be significantly elevated on PBMCs in demyelinated lesions in the CNS [31]. An increase in the expression of $\alpha 4 \beta 1$ on the cell surface may suggest increased migration of CD8+ T cells to the CNS. High levels of $\alpha 4 \beta 1$ on the CD8+ T cells in particular, in the naïve and EMRA subsets may indicate a reduced prevalence of soluble VCAM-1, IFN- $\gamma$, and TNF [32]. Soluble VCAM-1 is known to suppress the function of $\alpha 4 \beta 1$ under normal physiological concentration, while in the CFS/ME patients decreased expression of CD49d/CD29 on EM CD8+ T cells may indicate reduced migration of effector cells to sites of inflammation. CD127 is the receptor for IL7 and is an important marker for $\mathrm{T}$ cell maturation and function. Binding of IL-7 to this receptor is a vital component in the release of granzymes resulting in demyelination [33]. In MS polymorphism in the CD127 gene sequence is an associated risk factor [34]. CD127 has four different haplotypes. Haplotype 1 results in the production of enormous amounts of sCD127; haplotype 2 is associated with a less soluble form of sCD127 due to low levels of exon 6 splicing and is associated with a lower risk of MS [35]. Homozygosity for haplotype 


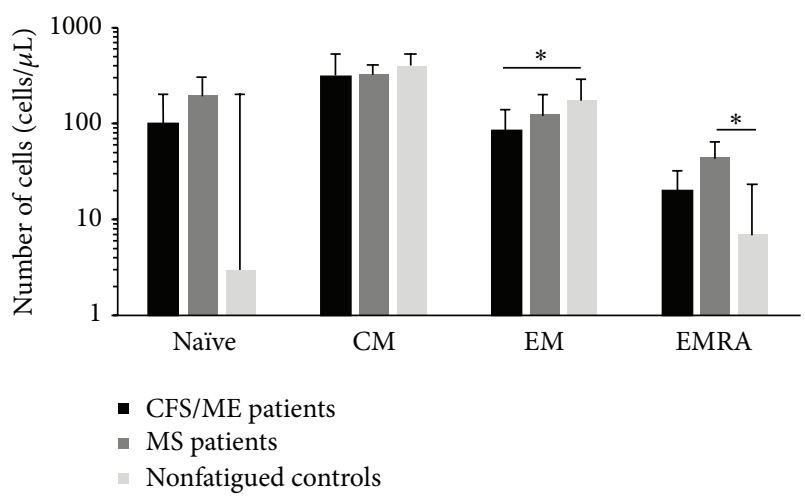

(a)

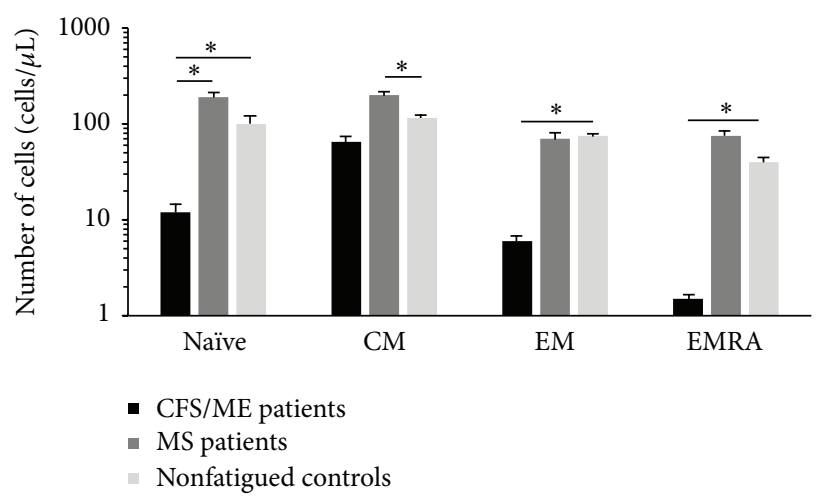

(b)

Figure 2: Expression of receptors in CFS/ME, MS, and nonfatigued controls. (a) CD49d/CD29 was reduced in EM subsets of CD8+ T cells in the CFS/ME patients but elevated in EMRA subsets of CD8+ T cells in MS patients compared to controls. (b) CD127 expression was reduced on naïve, EM, and EMRA subsets of CD8+ T cells in the CFS/ME patients but not CM subsets as CD127+ CD8+ T cells were evaluated in MS patients compared with controls. MS patients also demonstrated elevated naïve CD8 T cells compared with controls. Data is represented as median \pm SEM, where * represents $P<0.05$ (EM: effector memory, EMRA: effector memory RA, and CM: central memory).

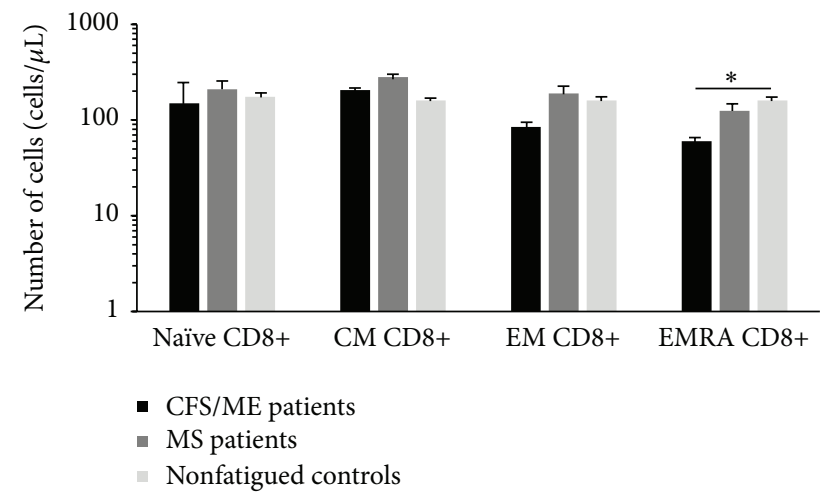

FIGURE 3: Expression of adhesion molecules on subsets of CD8+ $\mathrm{T}$ cells in CFS/ME, MS, and nonfatigued controls. Expression levels of PSGL-1 were reduced on EMRA CD8+ T cells in the $\mathrm{CFS} / \mathrm{ME}$ patients compared with the nonfatigued controls. Data is represented as \pm SEM, where $*$ represents $P<0.05$ (EM: effector memory, EMRA: effector memory RA, and CM: central memory).

4 increases the likelihood of MS [36]. It has been suggested that polymorphisms in CD127 and its cytokine IL-7 may be correlated with susceptibility to MS [37]. The MS group in this study demonstrated heightened levels of CD127+ on all subsets of $\mathrm{CD} 8+\mathrm{T}$ cells in comparison to the CFS/ME patients but this was only significant in the EMRA CD8+ T cells. Interestingly, IL-7 may inhibit the function of VCAM1 by binding to it and thus allowing the dominance of $\alpha 4 \beta 1$ integrin which facilitates the movement of $\mathrm{T}$ cells to the CNS. In the CNS, CD8+ T cell cytotoxic activity may be further enhanced by IL-7 resulting in an overabundance of granzymes and consequently increasing demyelination in the CNS $[38,39]$. The expression of CD127+ was decreased on most $\mathrm{CD} 8+\mathrm{T}$ cell subsets in the CFS/ME patients compared with both MS and controls. Reduced CD127 in the CFS/ME patients was corroborated with reduced $\alpha 4 \beta 1$ integrin confirming significant alterations in the migratory potential of the CD8+ T cells in CFS/ME patients. The exact role of CD127 on CD8+ T cells in CFS/ME is unclear though it has been suggested that reduced CD127 on exhausted CD8+ $\mathrm{T}$ cells might be responsible for the inability for $\mathrm{CD} 8+\mathrm{T}$ cells to suppress viral persistence [40]. CD8+ T cell exhaustion has been previously suggested in CFS/ME owing to the overwhelming levels of other exhaustion markers including PD1 and CD95 [24]. BTLA is another inhibitory coreceptor expressed on CD8+ T cell; similar to CD127, BTLA expression was increased in the MS patients compared to the CFS/ME patients. BTLA is known to bind to TNF receptor family member herpesvirus entry mediator (HVEM). This initiates a sequence of events involving phosphorylation of ITIM motifs and induction of phosphatases SHP-1 and SHP-2 [41]. BTLA and HVEM interact in either cis or trans configuration and this inhibits or activates NF- $\kappa$ B, respectively [42]. Increased expression of BTLA on naïve and CM CD8+ T cells may indicate suppression of $\mathrm{T}$ cell receptor signalling via $\mathrm{CD} 3$ and/or CD28 [43]. Additionally, BTLA interaction with HVEM promotes cell survival and memory generation of effector CD8+ T cells [44].

Previous research has indicated decreases in total CD8+ $\mathrm{T}$ cells in particularly EM and EMRA CD8+ T cells $[45,46]$. However, in the present study although total CD8+ EM and EMRA T cells were reduced in MS patients this was not statistically significant.

\section{Conclusions}

In summary, these preliminary findings provide new insight into the possibility of hyper activated inflammatory CD8+ T cell profile in untreated MS patients while CFS/ME patients may display an exhausted profile which permits viral prevalence and persistence. The above data may suggest that the differential expressions of receptors and adhesion molecules in MS patients are in response to imbalances in neuroimmune 
homeostasis. In comparison to CFS/ME patients, MS patients may have more severe immune dysregulation. Nevertheless it is likely that impairments in CD8+ $\mathrm{T}$ cells in CFS/ME patients relate to abnormal levels of adhesion and migratory molecules and these abnormalities may contribute to the persistent immune dysregulation observed and warrant further validation in a larger sample size.

\section{Abbreviations}

$\begin{array}{ll}\text { CD: } & \text { Cluster of differentiation } \\ \text { MS: } & \text { Multiple Sclerosis } \\ \text { CFS/ME: } & \text { Chronic Fatigue Syndrome/Myalgic } \\ & \text { Encephalomyelitis } \\ \text { CM: } & \text { Central memory } \\ \text { EM: } & \text { Effector memory } \\ \text { EMRA: } & \text { Effector memory cells expressing CD45RA } \\ \text { BTLA: } & \text { B- and T-lymphocyte attenuator } \\ \text { KLRG1: } & \text { Killer cell lectin-like receptor subfamily G } \\ & \text { member 1 } \\ \text { LAG3: } & \text { Lymphocyte-activation protein } 3 \\ \text { CTLA-4: } & \text { Cytotoxic T-lymphocyte-associated protein } 4 \\ \text { CCR7: } & \text { Chemokine receptor type } 7 \\ \text { CCR5: } & \text { Chemokine receptor type } 5 \\ \text { VCAM-1: } & \text { Vascular cell adhesion protein } 1 \\ \text { IFN- } \gamma: & \text { Interferon gamma } \\ \text { TNF: } & \text { Tumor necrosis factor } \\ \text { IL-7: } & \text { Interleukin-7 } \\ \text { CNS: } & \text { Central Nervous System } \\ \text { PD1: } & \text { Programmed cell death protein } 1 \\ \text { HVEM: } & \text { Herpes virus entry mediator } \\ \text { NF- } \kappa \text { B: } & \text { Nuclear factor kappa-light-chain enhancer of } \\ & \text { activated B cells. }\end{array}$

\section{Disclaimer}

The authors alone are responsible for the content and writing of the paper.

\section{Conflict of Interests}

The authors report no conflict of interests.

\section{Authors' Contribution}

Ekua W. Brenu, Thao Nguyen, and Samantha Johnston wrote the paper, Simon Broadley provided the MS patients and reviewed the paper, and Sonya Marshall-Gradisnik and Don Staines provided supervision and assisted with the writing of the paper.

\section{Acknowledgments}

The authors would like to acknowledge the National Centre for Neuroimmunology and Emerging Diseases, Alison Hunter Memorial Foundation, Mason Foundation (Grant no.
MA43120), and Queensland Government Science, Information Technology, Innovation and the Arts Smart Futures Fund (Grant no. 216702MRE) for their support.

\section{References}

[1] P. J. Jongen, D. Lehnick, J. Koeman et al., "Fatigue and healthrelated quality of life in relapsing-remitting multiple sclerosis after 2 years glatiramer acetate treatment are predicted by changes at 6 months: an observational multi-center study," Journal of Neurology, vol. 261, no. 8, pp. 1469-1476, 2014.

[2] M. H. Cameron, V. Peterson, E. A. Boudreau et al., "Fatigue is associated with poor sleep in people with multiple sclerosis and cognitive impairment," Multiple Sclerosis International, vol. 2014, Article ID 872732, 5 pages, 2014.

[3] A. A. Holland, D. Graves, B. M. Greenberg, and L. L. Harder, "Fatigue, emotional functioning, and executive dysfunction in pediatric multiple sclerosis," Child Neuropsychology, vol. 20, no. 1, pp. 71-85, 2014.

[4] H. Hildebrandt and P. Eling, "A longitudinal study on fatigue, depression, and their relation to neurocognition in multiple sclerosis," Journal of Clinical and Experimental Neuropsychology, vol. 36, no. 4, pp. 410-417, 2014.

[5] N. Razazian, N. Shokrian, A. Bostani, N. Moradian, and S. Tahmasebi, "Study of fatigue frequency and its association with sociodemographic and clinical variables in patients with multiple sclerosis," Neurosciences, vol. 19, no. 1, pp. 38-42, 2014.

[6] T. A.-Z. K. Gaber, W. W. Oo, and H. Ringrose, "Multiple sclerosis/chronic fatigue syndrome overlap: when two common disorders collide," NeuroRehabilitation, vol. 35, no. 3, pp. 529534, 2014.

[7] C. H. Polman, S. C. Reingold, B. Banwell et al., "Diagnostic criteria for multiple sclerosis: 2010 revisions to the McDonald criteria," Annals of Neurology, vol. 69, no. 2, pp. 292-302, 2011.

[8] W. I. McDonald, A. Compston, G. Edan et al., "Recommended diagnostic criteria for multiple sclerosis: guidelines from the International Panel on the diagnosis of multiple sclerosis," Annals of Neurology, vol. 50, no. 1, pp. 121-127, 2001.

[9] L. R. Barnden, B. Crouch, R. Kwiatek et al., "A brain MRI study of chronic fatigue syndrome: evidence of brainstem dysfunction and altered homeostasis," NMR in Biomedicine, vol. 24, no. 10, pp. 1302-1312, 2011.

[10] D. B. Cook, G. Lange, J. Deluca, and B. H. Natelson, "Relationship of brain MRI abnormalities and physical functional status in chronic fatigue syndrome," International Journal of Neuroscience, vol. 107, no. 1-2, pp. 1-6, 2001.

[11] Y. Nakatomi, K. Mizuno, A. Ishii et al., "Neuroinflammation in patients with chronic fatigue syndrome/myalgic encephalomyelitis: An11C-(R)-PK11195 PET study," Journal of Nuclear Medicine, vol. 55, no. 6, pp. 945-950, 2014.

[12] B. K. Puri, P. M. Jakeman, M. Agour et al., "Regional grey and white matter volumetric changes in myalgic encephalomyelitis (chronic fatigue syndrome): a voxel-based morphometry $3 \mathrm{~T}$ MRI study," British Journal of Radiology, vol. 85, no. 1015, pp. e270-e273, 2012.

[13] G. Morris and M. Maes, "Myalgic encephalomyelitis/chronic fatigue syndrome and encephalomyelitis disseminata/multiple sclerosis show remarkable levels of similarity in phenomenology and neuroimmune characteristics," BMC Medicine, vol. 11, no. 1, article 205, 2013. 
[14] C. Chanvillard, R. F. Jacolik, C. Infante-Duarte, and R. C. Nayak, "The role of natural killer cells in multiple sclerosis and their therapeutic implications," Frontiers in Immunology, vol. 4, article 63, 2013.

[15] G. Kaur, J. Trowsdale, and L. Fugger, "Natural killer cells and their receptors in multiple sclerosis," Brain, vol. 136, no. 9, pp. 2657-2676, 2013.

[16] E. W. Brenu, T. K. Huth, S. L. Hardcastle et al., "Role of adaptive and innate immune cells in chronic fatigue syndrome/myalgic encephalomyelitis," International Immunology, vol. 26, no. 4, Article ID dxt068, pp. 233-242, 2014.

[17] E. W. Brenu, M. L. van Driel, D. R. Staines et al., "Longitudinal investigation of natural killer cells and cytokines in chronic fatigue syndrome/myalgic encephalomyelitis," Journal of Translational Medicine, vol. 10, no. 1, article 88, 2012.

[18] E. W. Brenu, M. L. van Driel, D. R. Staines et al., "Immunological abnormalities as potential biomarkers in Chronic Fatigue Syndrome/Myalgic Encephalomyelitis," Journal of Translational Medicine, vol. 9, article 81, 2011.

[19] D. E. Lowther and D. A. Hafler, "Regulatory T cells in the central nervous system," Immunological Reviews, vol. 248, no. 1, pp. 156169, 2012.

[20] L. Cervantes-Barragán, S. Firner, I. Bechmann et al., "Regulatory $\mathrm{T}$ cells selectively preserve immune privilege of selfantigens during viral central nervous system infection," The Journal of Immunology, vol. 188, no. 8, pp. 3678-3685, 2012.

[21] S. Markovic-Plese, C. Pinilla, and R. Martin, "The initiation of the autoimmune response in multiple sclerosis," Clinical Neurology and Neurosurgery, vol. 106, no. 3, pp. 218-222, 2004.

[22] R. S. Lopez-Diego and H. L. Weiner, "Novel therapeutic strategies for multiple sclerosis-a multifaceted adversary," Nature Reviews Drug Discovery, vol. 7, no. 11, pp. 909-925, 2008.

[23] S. Sinha, F. R. Itani, and N. J. Karandikar, "Immune regulation of multiple sclerosis by CD8+ T cells," Immunologic Research, vol. 59, no. 1-3, pp. 254-265, 2014.

[24] M. Curriu, J. Carrillo, M. Massanella et al., "Screening NK-, Band T-cell phenotype and function in patients suffering from Chronic Fatigue Syndrome," Journal of Translational Medicine, vol. 11, article 68, 2013.

[25] B. M. Carruthers, M. I. Van de Sande, K. L. De Meirleir et al., "Myalgic encephalomyelitis: International Consensus Criteria," Journal of Internal Medicine, vol. 270, no. 4, pp. 327-338, 2011.

[26] M. M. Brown, D. S. Bell, L. A. Jason, C. Christos, and D. E. Bell, "Understanding long-term outcomes of chronic fatigue syndrome," Journal of Clinical Psychology, vol. 68, no. 9, pp. 1028-1035, 2012.

[27] I. Kister, E. Chamot, A. R. Salter, G. R. Cutter, T. E. Bacon, and J. Herbert, "Disability in multiple sclerosis: a reference for patients and clinicians," Neurology, vol. 80, no. 11, pp. 1018-1024, 2013.

[28] S. L. Hardcastle, E. Brenu, S. Johnston et al., "Analysis of the relationship between immune dysfunction and symptom severity in patients with chronic fatigue syndrome/myalgic encephalomyelitis (CFS/ME)," Journal of Clinical \& Cellular Immunology, vol. 5, article 190, 2014.

[29] B. Bahbouhi, L. Berthelot, S. Pettré et al., "Peripheral blood $\mathrm{CD}^{+} \mathrm{T}$ lymphocytes from multiple sclerosis patients are characterized by higher PSGL-1 expression and transmigration capacity across a human blood-brain barrier-derived endothelial cell line," Journal of Leukocyte Biology, vol. 86, no. 5, pp. 1049-1063, 2009.
[30] L. Battistini, L. Piccio, B. Rossi et al., " $\mathrm{CD} 8^{+} \mathrm{T}$ cells from patients with acute multiple sclerosis display selective increase of adhesiveness in brain venules: a critical role for P-selectin glycoprotein ligand-1," Blood, vol. 101, no. 12, pp. 4775-4782, 2003.

[31] L. Bö, J. W. Peterson, S. Mørk et al., "Distribution of immunoglobulin superfamily members ICAM-1, -2, -3, and the $\beta 2$ integrin LFA-1 in multiple sclerosis lesions," Journal of Neuropathology and Experimental Neurology, vol. 55, no. 10, pp. 1060-1072, 1996.

[32] B. A. Kallmann, V. Hummel, T. Lindenlaub, K. Ruprecht, K. V. Toyka, and P. Rieckmann, "Cytokine-induced modulation of cellular adhesion to human cerebral endothelial cells is mediated by soluble vascular cell adhesion molecule-1," Brain, vol. 123, no. 4, pp. 687-697, 2000.

[33] R. Mazzucchelli and S. K. Durum, "Interleukin-7 receptor expression: intelligent design," Nature Reviews Immunology, vol. 7, no. 2, pp. 144-154, 2007.

[34] S. G. Gregory, S. Schmidt, P. Seth et al., "Interleukin 7 receptor $\alpha$ chain (IL7R) shows allelic and functional association with multiple sclerosis," Nature Genetics, vol. 39, no. 9, pp. 1083-1091, 2007.

[35] F. C. McKay, L. I. Swain, S. D. Schibeci et al., "Haplotypes of the interleukin 7 receptor alpha gene are correlated with altered expression in whole blood cells in multiple sclerosis," Genes and Immunity, vol. 9, no. 1, pp. 1-6, 2008.

[36] E. Hoe, F. C. McKay, S. D. Schibeci et al., "Functionally significant differences in expression of disease-associated IL-7 receptor $\alpha$ haplotypes in CD4 T cells and dendritic cells," The Journal of Immunology, vol. 184, no. 5, pp. 2512-2517, 2010.

[37] K. L. Kreft, E. Verbraak, A. F. Wierenga-Wolf et al., "Decreased systemic IL-7 and soluble IL-7Ralpha in multiple sclerosis patients," Genes and Immunity, vol. 13, no. 7, pp. 587-592, 2012.

[38] C. Malmeström, J. Lycke, S. Haghighi et al., "Relapses in multiple sclerosis are associated with increased $\mathrm{CD}^{+} \mathrm{T}$-cell mediated cytotoxicity in CSF," Journal of Neuroimmunology, vol. 196, no. 1-2, pp. 159-165, 2008.

[39] K. L. Kreft, E. Verbraak, A. F. Wierenga-Wolf et al., "The IL-7R $\alpha$ pathway is quantitatively and functionally altered in CD8 T cells in multiple sclerosis," The Journal of Immunology, vol. 188, no. 4, pp. 1874-1883, 2012.

[40] M. Paiardini, B. Cervasi, H. Albrecht et al., "Loss of CD127 expression defines an expansion of effector CD8+ T cells in HIV-infected individuals," Journal of Immunology, vol. 174, no. 5, pp. 2900-2909, 2005.

[41] J. R. Sedy, M. Gavrieli, K. G. Potter et al., "B and T lymphocyte attenuator regulates $\mathrm{T}$ cell activation through interaction with herpesvirus entry mediator," Nature Immunology, vol. 6, no. 1, pp. 90-98, 2005.

[42] T. C. Cheung, L. M. Oborne, M. W. Steinberg et al., "T cell intrinsic heterodimeric complexes between HVEM and BTLA determine receptivity to the surrounding microenvironment," The Journal of Immunology, vol. 183, no. 11, pp. 7286-7296, 2009.

[43] A. C. Vendel, J. Calemine-Fenaux, A. Izrael-Tomasevic, V. Chauhan, D. Arnott, and D. L. Eaton, "B and T lymphocyte attenuator regulates B cell receptor signaling by targeting Syk and BLNK," The Journal of Immunology, vol. 182, no. 3, pp. 1509$1517,2009$.

[44] M. W. Steinberg, Y. Huang, Y. Wang-Zhu, C. F. Ware, H. Cheroutre, and M. Kronenberg, "BTLA interaction with HVEM expressed on $\mathrm{CD}^{+} \mathrm{T}$ cells promotes survival and memory 
generation in response to a bacterial infection," PLoS ONE, vol. 8, no. 10, Article ID e77992, 2013.

[45] M. P. Pender, P. A. Csurhes, C. M. Pfluger, and S. R. Burrows, "Deficiency of $\mathrm{CD}^{+}$effector memory $\mathrm{T}$ cells is an early and persistent feature of multiple sclerosis," Multiple Sclerosis Journal, vol. 20, no. 14, pp. 1825-1832, 2014.

[46] M. P. Pender, P. A. Csurhes, C. M. M. Pfluger, and S. R. Burrows, "CD8 T cell deficiency impairs control of EpsteineBarr virus and worsens with age in multiple sclerosis," Journal of Neurology, Neurosurgery and Psychiatry, vol. 83, no. 3, pp. 353354, 2012. 


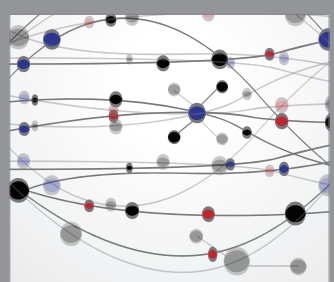

The Scientific World Journal
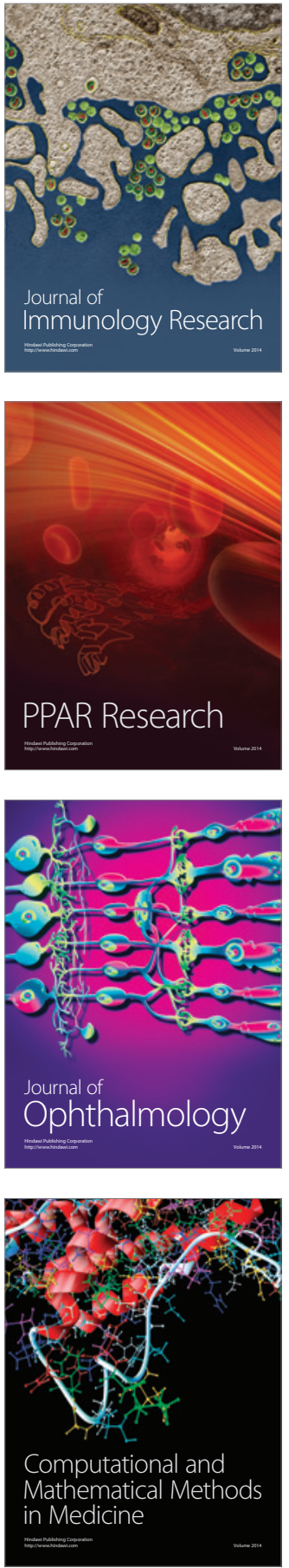

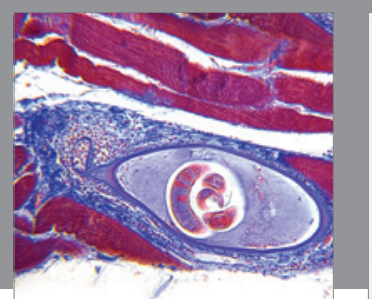

Gastroenterology Research and Practice

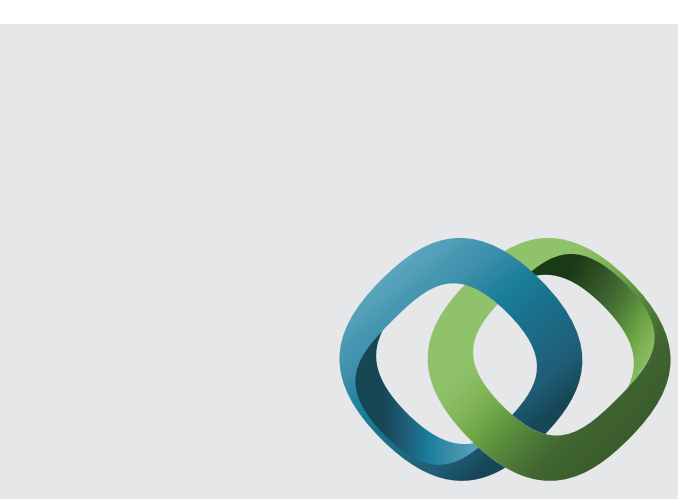

\section{Hindawi}

Submit your manuscripts at

http://www.hindawi.com
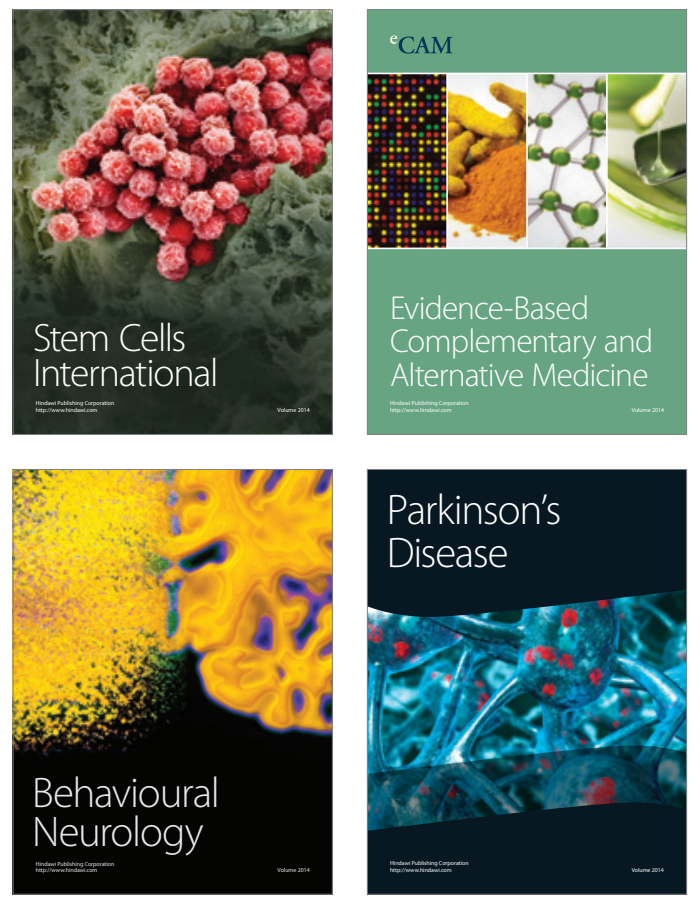
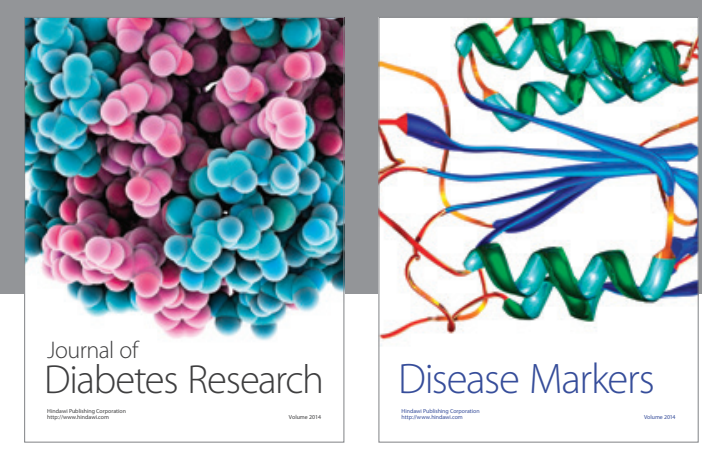

Disease Markers
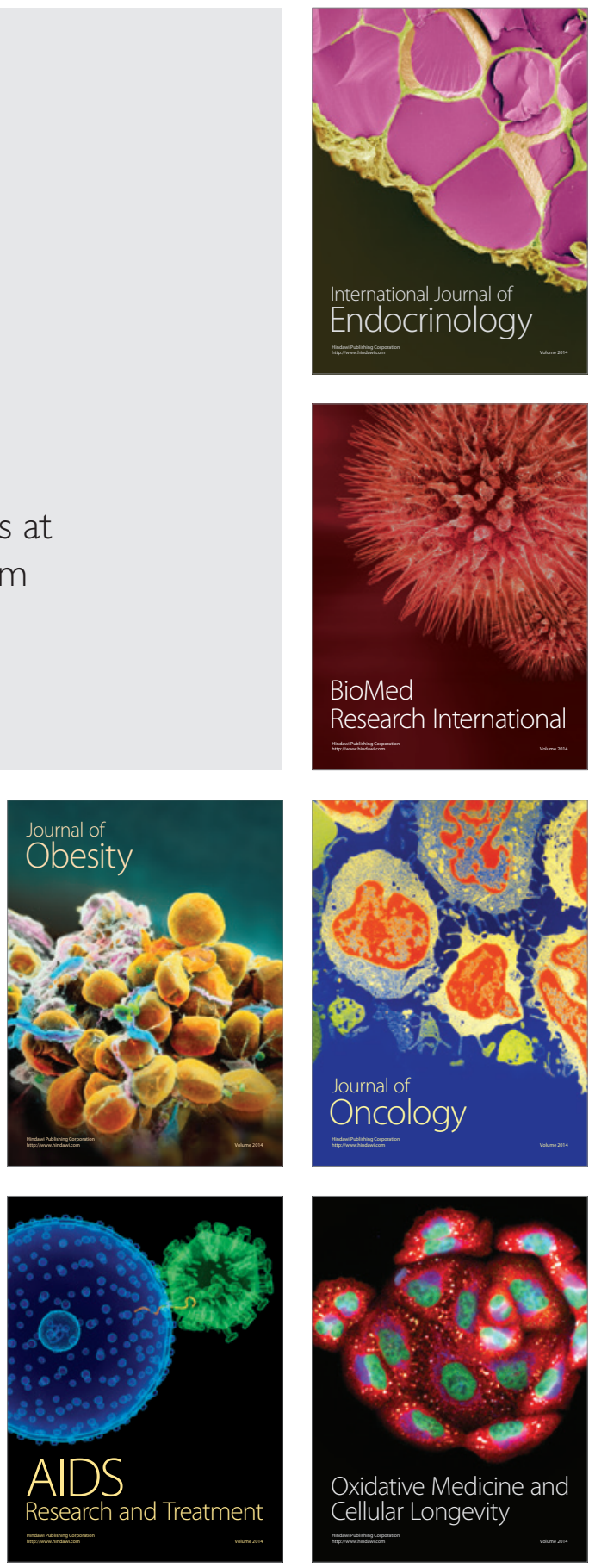